\title{
SOLUTE-SOLVENT INTERACTION IN AQUEOUS SOLUTIONS STUDIED BY OPTICAL KERR EFFECT
}

\author{
Z. Blaszczak and M. Farhoud* \\ Quantum Electronics Laboratory, Institute of Physics, A. Mickiewicz University \\ Grunwaldzka 6, 60-780 Poznań, Poland \\ (Received October 10, 1995; in final form November 22, 1996)
}

Solute-solvent interactions were studied by a method based on laser induced optical birefringence in aqueous solutions. The paper reports results of the optical Kerr constant measurements for binary liquid mixtures of aqueous solution of methanol, ethanol, $n$-propanol and acetone at room temperature in a wide range of concentrations. At $50 \%$ concentration of the above mentioned alcohols, the temperature dependence of the laser induced optical birefringence was also measured in the range from $283 \mathrm{~K}$ to $315 \mathrm{~K}$. These data together with the density and refractive index were used to calculate the optical and specific Kerr constants. Moreover, these data enabled the calculation of the anisotropic factors, optical anisotropy and orientation function of the substances studied.

PACS numbers: 42.25.Lc, 42.65.An

\section{Introduction}

In a liquid placed in a uniform electric field the optical birefringence is induced which is known as the electric Kerr effect (EKE). Kerr constant, which describes microscopic properties of the medium, is considered as a very sensitive indicator of molecular associations in liquids and solutions. The properties of liquid mixtures have been investigated by many groups using different methods [1]. Patz and Rätzsch [2], Pyzuk et al. [3], the group working at the Kharkov Polytechnical Institute [4] and Piazza et al. [5] have measured electric Kerr constant for several binary liquid mixtures.

The use of water as a solvent for the measurement of electric Kerr effect possesses some problems. Firstly, an electric field of short duration has to be generated across a conducting solution by a well-defined high voltage. Secondly, there is a formidable task of analysing the Kerr effect data in terms of intrinsic molecular properties and specific solvent effects. These difficulties can be overcome

*Present address: Physics Department, Faculty of Science, Alexandria University, P.O. Box 21511, Alexandria, Egypt. 
by electrodeless application of the electric field to the medium, that is by using an electric field of optical frequency of a powerful laser beam. In that case optical birefringence (high frequency Kerr effect) takes place. This effect, which is also called optical Kerr effect (OKE), has been the subject of numerous theoretical and experimental studies in nanosecond [6], picosecond [7] and femtosecond $[8,9]$ time domains. The concentration and temperature dependence of the optical Kerr effect in different solutions $[10,11]$ has been also studied. Particular attention has been paid to OKE in alcohols in nanosecond time domain. For all the alcohols studied, a linear relationship between the carbon chain length and optical Kerr constant was found [12,13]. The results reported by Ho and Alfano [7], who measured the picosecond optical Kerr response of five alcohols, could also be interpreted as supporting this finding. For longer chain alcohols they noted abnormally long relaxation times consistent with the reorientation phenomena involved.

OKE is interesting for a number of reasons. Firstly, it is a direct measure of the induced polarizability of molecules as the applied field frequency is too high for permanent dipolar mechanisms to participate. Knowledge of this polarizability leads directly to the details of the atomic bonding structure. Secondly, by inducing orientational order it offers a means of measuring fast relaxation processes as well as the size of small particles and molecules using fast switched laser pulses. Thirdly, OKE can be a potential mechanism for optically activated molecular switches. Moreover, the phenomenon is a third-order nonlinear optical effect and as such provides a method for measurement of the third-order nonlinear susceptibility (polarizability).

Moreover, the OKE method is particularly advantageous when compared with EKE studies of aqueous solutions because the application of electric field without electrodes eliminates many undesirable effects induced by the electric current flowing through aqueous solutions.

Optical birefringence " $\delta n$ " induced in the medium by a strong optical field of amplitude " $E$ " is defined as

$$
\delta n=B_{0} \lambda E^{2}
$$

where $B_{\circ}$ is the optical Kerr constant and $\lambda$ is the wavelength of the analysing beam.

From the Peterlin-Stuart theory, the specific Kerr constant $B_{\mathrm{sp}}$ of a solution affected by an electric field is given by the equation [14]:

$$
B_{\mathrm{sp}}=\frac{2 \pi\left(g_{1}-g_{2}\right)}{15 n^{2}}(P+Q),
$$

where $n$ is the refractive index of the solute, $g_{1}$ and $g_{2}$ are the anisotropic factors along the parallel and perpendicular directions relative to the optical field; $P$ and $Q$ - orientation parameters; $P=(\mu / k T)^{2}$ and $Q=(\delta \alpha) / k T, f$ - orientation function; $f=P+Q,(\delta \alpha)$ - optical anisotropy, $\mu-$ effective induced dipole moment, $k-$ Boltzmann constant, $T$ - absolute temperature; also

$$
\delta \alpha=4 \pi \epsilon_{0} \nu\left(g_{1}-g_{2}\right) \text {, }
$$

where $\epsilon_{0}$ - permittivity of free space, $\nu$ - partial volume.

At optical frequencies, the processes of permanent dipole orientation cannot take place and the term $P$ of Eq. (2) is ineffective. Since the value of $B_{\mathrm{sp}}$ is known, the values of $\delta g$ and other parameters could be calculated. 
Molecular properties of the medium are described by the molar optical Kerr constant $B_{\mathrm{m}}\left(\lambda_{\mathrm{a}}, \lambda_{\mathrm{i}}\right)$, dependent on parameters characterizing the atoms, molecules and on the molecular mechanism of birefringence. In the case of dense media molar and optical Kerr constants are related as follows [15]:

$$
B_{\mathrm{m}}\left(\lambda_{\mathrm{a}}, \lambda_{\mathrm{i}}\right)=54 n_{\mathrm{a}} \lambda_{\mathrm{a}} M\left(n_{\mathrm{a}}^{2}+2\right)^{-2} d^{-1} B_{0},
$$

where $n_{\mathrm{a}}$ and $n_{\mathrm{i}}$ are refractive indices for the analysing and the inducing beam, respectively, $M$ is the molecular weight of the sample under test, $d-$ its density which equals $M / V_{\mathbf{M}}$, in which $V_{\mathbf{M}}$ is the molar volume.

From the point of view of the molecular mechanisms which on the macroscopic scale lead to the appearance of the induced birefringence in the medium, the constant $B_{\mathrm{m}}$ may be written as a sum of three terms [15]:

$$
B_{\mathrm{m}}\left(\lambda_{\mathrm{a}}, \lambda_{\mathrm{i}}\right)=B_{\mathrm{m}}^{\mathrm{NL}}\left(\lambda_{\mathrm{a}}, \lambda_{\mathrm{i}}\right)+B_{\mathrm{m}}^{\mathrm{ORI}}\left(\lambda_{\mathrm{a}}, \lambda_{\mathrm{i}}\right)+B_{\mathrm{m}}^{\mathrm{RED}}\left(\lambda_{\mathrm{a}}, \lambda_{\mathrm{i}}\right)
$$

where $B_{\mathrm{m}}^{\mathrm{NL}}$ is the nonlinear molar optical Kerr constant which describes the contribution to OKE due to deformation of the electron cloud of an atom or molecule in an optical field, $B_{\mathrm{m}}^{\mathrm{ORI}}$ represents the contribution to OKE due to the orientation of the molecules and $B_{\mathrm{m}}^{\mathrm{RED}}$ represents the redistribution of the atoms and molecules in the field. Given the above definitions of $B_{\mathrm{m}}^{\mathrm{NL}}, \mathrm{B}_{\mathrm{m}}^{\mathrm{ORI}}$ and $B_{\mathrm{m}}^{\mathrm{RED}}$, Eq. (4) could be written [16]:

$$
B_{\mathrm{m}}\left(\lambda_{\mathrm{a}}, \lambda_{\mathrm{i}}\right)=\frac{4}{9} \pi N_{\mathrm{A}} c+\frac{4 \pi N_{\mathrm{A}}}{45 k T} \Gamma^{2}\left(\lambda_{\mathrm{a}}, \lambda_{\mathrm{i}}\right)
$$

where $c$ is the mean nonlinear polarizability, $N_{\mathrm{A}}$ is the Avogadro number, $k$ is the Boltzmann constant, $T$ - the absolute temperature, and $\Gamma$ - the effective optical anisotropy characterizing the optical anisotropy of a molecule immersed in a liquid. Taking into account the properties of the molecules and their interactions, as well as the temperature dependence of the molar Kerr constants, Eq. (5) becomes

$$
B_{\mathrm{m}}\left(\lambda_{\mathrm{a}}, \lambda_{\mathrm{i}}\right)=a+b T^{-1},
$$

where $a=(4 \pi / 9) N_{\mathrm{A}} c$ and $b=\left(4 \pi N_{\mathrm{A}} / 45 k\right) \Gamma^{2}\left(\lambda_{\mathrm{a}}, \lambda_{\mathrm{i}}\right)$. The constants $a$ and $b$ can be obtained from the dependence of $B_{\mathrm{m}}$ on the inverse temperature, hence the values of $c$ and $\Gamma^{2}$ can be derived.

To the best of our knowledge, this is the first report on the concentration and temperature dependence of optically induced birefringence in aqueous solution of methanol, ethanol, $n$-propanol and acetone at the wavelength $488.0 \mathrm{~nm}$. From these data, the anisotropic factors, optical anisotropy and orientation function were calculated.

\section{Experimental setup}

The setup for OKE measurements used in the experiment was constructed at our laboratory (Fig. 1). OKE was induced with a beam of a ruby laser $\left(\lambda_{\mathrm{i}}=\right.$ $694.3 \mathrm{~nm})$ generating nanosecond pulses $\left(\tau_{\text {pulse }}=10 \mathrm{~ns}\right.$, power $\left.20 \mathrm{MW}\right)$ and analysed with a beam of a continuously operating argon laser $\left(\lambda_{\mathrm{a}}=488.0 \mathrm{~nm}\right.$, about $100 \mathrm{~mW}$ in power). Both lasers worked in the fundamental mode $\left(\mathrm{TEM}_{00}\right)$ and in one (or at most two - in the case of the ruby laser) longitudinal mode. The longitudinal mode of the argon laser was selected by a Fabry-Pérot etalon made by $\mathrm{C}$. Zeiss Jena, mounted in the optical resonator. For selection of modes of the ruby laser we used a special three-plate Fabry-Pérot etalon, designed and constructed 




Fig. 1. Diagram of the experimental setup.

in our laboratory, placed in the optical resonator (of about $30 \mathrm{~cm}$ in length) of the ruby laser. The temporal pulse profile of the inducing beam was nearly Gaussian. The linearly polarized laser beams propagated exactly coaxially in the liquids studied. The polarization of both beams was corrected with the help of Glan polarizers fixing an angle of $45^{\circ}$ between their electric vectors at the input to the cuvette which provided the optimum geometry of measurement [17]. The power of the ruby laser beam was recorded by a calibrated fast photodiode BPDP-41. An analyser crossed with the polarizer of the analysing beam prevented this beam from falling into a M12FQ51 photomultiplier by which the effect was recorded. During the flash of the ruby laser the medium under investigation became optically birefringent. This means that a part of the analysing beam came through the analyser and as a consequence the photomultiplier recorded a pulse of the analysing beam proportional to the magnitude of the optically induced birefringence. Electric pulses from the photodiode and the photomultiplier (both working in their linear range) were simultaneously observed and recorded by an oscillograph S8-12A with a memory. The applied method of coincidence enhanced the sensitivity of the measuring system. After each series of measurement a calibration curve was taken with the help of $80 \mu$ s pulses of the analysing beam modulated mechanically. This procedure gave the dependence of the height of the pulse on the oscilloscope screen on the angle $\delta$ of rotation of the analyser which could be read out with an accuracy of $0.05^{\circ}$ and which was a measure of the liquid's birefringence $\left(B_{\circ}=\delta / \sqrt{2} \pi l E^{2}\right.$ (Ref. [17]).

It is known that determination of the absolute value of the optical Kerr constant requires the knowledge of the power density of the inducing beam in the studied medium, which is necessary to calculate the intensity of the optical field. To be able to establish this power density, the space and time characteristics of the inducing laser emission must be found. This is not an easy task even when a laser works in the fundamental mode when the beam is Gaussian. Therefore 
most often relative measurements are performed assuming a certain liquid as a reference standard. As such a standard usually benzene is chosen for which there is a relatively large body of literature data. Consequently having assembled and adjusted the apparatus we performed scrupulous and precise measurements of the absolute Kerr constant values for benzene obtaining results which were in good agreement with the literature data. Moreover, we performed systematic studies for toluene and water which were also treated as reference standards in subsequent studies.

The Kerr cell consisted of a $5 \mathrm{~cm}$ long glass cuvette closed off with free birefringence optical windows. The temperature of the solution used was controlled by MLW-10U type ultrathermostat with an accuracy of $\pm 0.1^{\circ} \mathrm{C}$. The principle and details of the setup were published elsewhere [18].

The spectrally or analytically pure liquids were produced by International Enzymes Limited. Different mixtures of organic solvents with double distilled water from 1-100 wt.\% were prepared using an electric balance (Mettler $\mathrm{H} 80$ ) with an accuracy $\pm 0.1 \mathrm{mg}$.

Additionally (using a Pulfrich refractometer), measurements of the mixture refractive indices $n_{\mathrm{i}}\left(\right.$ for $\lambda_{\mathrm{i}}=694.3 \mathrm{~nm}$ ) and $n_{\mathrm{a}}$ (for $\lambda_{\mathrm{a}}=488.0 \mathrm{~nm}$ ) and density as functions of concentration and temperature were carried out.

\section{Results and discussion}

The work was undertaken to study the concentration and temperature dependences of the optical Kerr effect of aqueous solutions of methanol, ethanol, $n$-propanol and acetone. As has been mentioned above, usually relative measurements of the optical Kerr constant are performed. In such a case the magnitude of the optical Kerr effect in the studied substances should be close to that of the reference standard, as only then the geometries of the measurements are comparable. Therefore measurements of the optical Kerr constant were performed relative to double-distilled water which was taken as a reference standard. The relative Kerr constant $B_{\mathrm{r}}$ is given by the relation [17]:

$$
B_{\mathrm{r}}=\frac{B_{\mathrm{s}}}{B_{\mathrm{w}}}=\frac{n_{\mathrm{i}, \mathrm{s}} \beta_{\mathrm{s}} A_{\mathrm{i}, \mathrm{w}}}{n_{\mathrm{i}, \mathrm{w}} \beta_{\mathrm{w}} A_{\mathrm{i}}, \mathrm{s}},
$$

where $\mathrm{w}$ and $\mathrm{s}$ refer to water and the solution, $n_{\mathrm{i}}$ is the refractive index of the inducing beam while $\beta$ are the angles of rotation of the analyser corresponding to the amplitudes of the OKE pulses induced by the laser beam of constant power, $A$ is the apparatus constant. The value of $B_{\mathfrak{r}}$ for each point, as calculated by the least squares method, is a statistical mean of about thirty measurements. The error in determination of the values of $B_{\mathrm{r}}$ was less than $\pm 10 \%$.

OKE measurements were performed using double-distilled water as the reference liquid. At room temperature, the optical Kerr constant $B_{\circ}$ determined for water was $4.21 \times 10^{-16}$ [SI] [13] ( $B_{\mathrm{o}}^{\text {benzene }}=70.2 \times 10^{-16}$ [SI], Ref. [18]), at a wavelength of $488.0 \mathrm{~nm}$. The obtained relative value of the optical Kerr constant $B_{\mathrm{r}}$ (relative to water), refractive indices $n_{\mathrm{a}}, n_{\mathrm{i}}$ and density $d$ of the aqueous solutions of methanol, ethanol, $n$-propanol and acetone at room temperature $(295 \mathrm{~K})$ in a wide range of concentrations are collected in Table $I$. Then the absolute value of the optical Kerr constant $B_{\mathrm{o}}$ as a function of concentration was calculated for all aqueous 
solutions and is also presented in Table I. Figure 2 shows the relation between the absolute value of optical Kerr constant and the volume fraction for aqueous solutions of methanol, ethanol, $n$-propanol and acetone. The specific Kerr constant $B_{\mathrm{sp}}$ was calculated from the slope of the straight lines in Fig. 2. It can be seen that $B_{0}$ values (Fig. 2) increase with increasing concentration of ethanol, $n$-propanol and acetone and decrease with increasing concentration of methanol. The values of $B_{0}$ can be ordered as follows: methanol $<$ water $<$ ethanol $<n$-propanol $<$ acetone. This order is consistent with the results of paper [11] and moreover with the orientation function $f$ of these solutions as shown in Table II. This table includes also the partial volume of the solute, anisotropic factors, optical anisotropy and the orientation function which is calculated from Eq. (2).

An analysis of the concentration dependences can lead to the conclusion that the anisotropic factor and optical anisotropy are independent of the partial volume of the molecules. As it is known, molecular associations in aqueous solutions occur due to hydrogen bonding interactions. This behaviour explains different rate of change of $B_{\circ}$ values with concentration for that system. From our previous study it is well known [19] that molecular interactions decrease the value of the optical Kerr constant. Therefore one can conclude that molecular interactions in acetone solutions are evidently weaker than in the investigated solutions of alcohols.

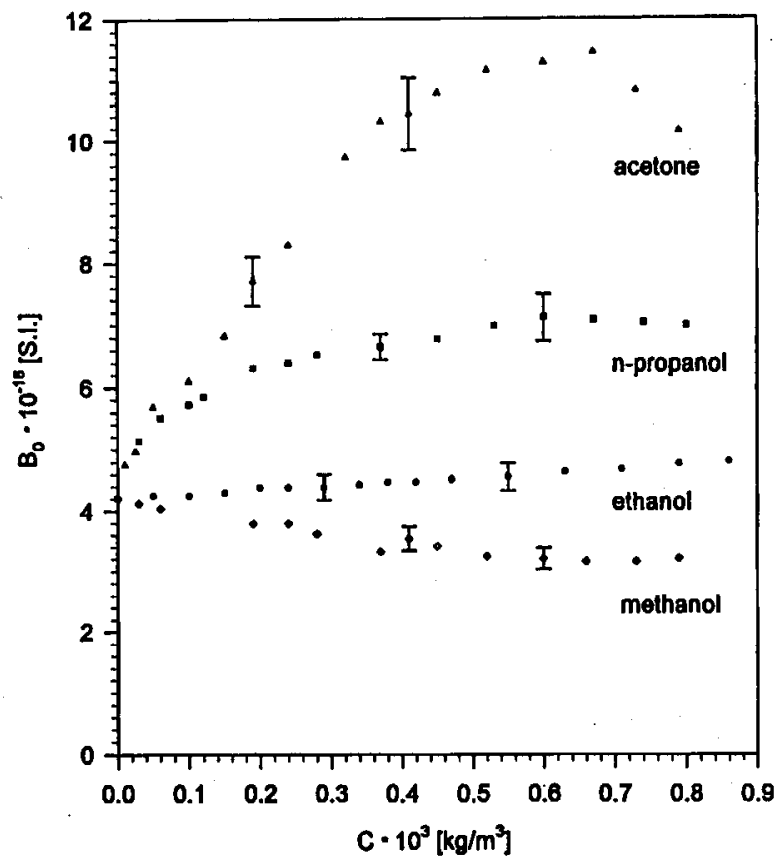

Fig. 2. The absolute values of optical Kerr constant for aqueous solutions versus volume fraction. 
TABLE I

The values of refractive indices $n_{\mathrm{a}}$ and $n_{\mathrm{i}}$, density, relative and absolute Kerr constants $B_{\mathrm{r}}$ and $B_{\mathrm{o}}$ as function of the volume fraction $C_{\nu}$ in aqueous solutions.

\begin{tabular}{c|l|l|c|c|c}
\hline \hline $\begin{array}{c}C_{\nu} \times 10^{3} \\
{\left[\mathrm{~kg} / \mathrm{m}^{3}\right]}\end{array}$ & \multicolumn{1}{c|}{$n_{\mathrm{a}}$} & \multicolumn{1}{c}{$n_{\mathrm{i}}$} & $\begin{array}{c}d \\
{\left[\mathrm{~kg} / \mathrm{m}^{3}\right]}\end{array}$ & $\begin{array}{c}B_{\mathrm{r}} \\
\text { to } \mathrm{H}_{2} \mathrm{O}\end{array}$ & $\begin{array}{c}B_{\mathrm{\circ}} \times 10^{-16} \\
{[\mathrm{SI}]}\end{array}$ \\
\hline \multicolumn{6}{c}{1. For methanol } \\
\hline 0.00 & 1.3367 & 1.3303 & 998.4 & 1.00 & 4.21 \\
0.03 & 1.3375 & 1.3311 & 991.8 & 0.98 & 4.13 \\
0.06 & 1.3384 & 1.3320 & 985.2 & 0.96 & 4.04 \\
0.19 & 1.3421 & 1.3357 & 956.1 & 0.90 & 3.79 \\
0.28 & 1.3435 & 1.3371 & 945.5 & 0.90 & 3.78 \\
0.37 & 1.3448 & 1.3384 & 935.0 & 0.86 & 3.62 \\
0.41 & 1.3489 & 1.3412 & 913.8 & 0.79 & 3.33 \\
0.45 & 1.3503 & 1.3439 & 903.2 & 0.84 & 3.54 \\
0.52 & 1.3530 & 1.3466 & 871.5 & 0.77 & 3.41 \\
0.60 & 1.3558 & 1.3494 & 855.6 & 0.76 & 3.24 \\
0.66 & 1.3585 & 1.3521 & 829.2 & 0.75 & 3.16 \\
0.73 & 1.3612 & 1.3548 & 808.0 & 0.75 & 3.16 \\
0.79 & 1.3640 & 1.3476 & 786.6 & 0.76 & 3.20 \\
\hline \multicolumn{6}{c}{2.51} \\
\hline 0.00 & 1.3331 & 1.329 & 998.4 & 1.0 & 4.21 \\
0.05 & 1.3403 & 1.3337 & 996.4 & 1.01 & 4.24 \\
0.10 & 1.343 & 1.3343 & 984.7 & 1.01 & 4.25 \\
0.15 & 1.3457 & 1.3389 & 978.6 & 1.02 & 4.3 \\
0.20 & 1.3483 & 1.3416 & 973.6 & 1.04 & 4.33 \\
0.24 & 1.351 & 1.3442 & 968.8 & 1.04 & 4.38 \\
0.29 & 1.3537 & 1.3469 & 962.2 & 1.04 & 4.39 \\
0.34 & 1.3563 & 1.3495 & 955.6 & 1.05 & 4.42 \\
0.38 & 1.359 & 1.3522 & 948.0 & 1.06 & 4.45 \\
0.42 & 1.3617 & 1.3548 & 939.5 & 1.06 & 4.47 \\
0.47 & 1.3643 & 1.3575 & 930.1 & 1.07 & 4.51 \\
0.55 & 1.3696 & 1.3627 & 916.6 & 1.08 & 4.57 \\
0.63 & 1.3749 & 1.368 & 902.0 & 1.1 & 4.63 \\
0.71 & 1.3802 & 1.3733 & 887.3 & 1.11 & 4.68 \\
0.79 & 1.3856 & 1.3785 & 872.7 & 1.13 & 4.76 \\
0.86 & 1.3909 & 1.3838 & 858.0 & 1.14 & 4.8 \\
\end{tabular}


TABLE I (cont.)

\begin{tabular}{c|c|c|c|c|c}
\hline \hline $\begin{array}{c}C_{\nu} \times 10^{3} \\
{\left[\mathrm{~kg} / \mathrm{m}^{3}\right]}\end{array}$ & $n_{\mathrm{a}}$ & $n_{\mathrm{i}}$ & $\begin{array}{c}d \\
{\left[\mathrm{~kg} / \mathrm{m}^{3}\right]}\end{array}$ & $\begin{array}{c}B_{\mathrm{r}} \\
\text { to } \mathrm{H}_{2} \mathrm{O}\end{array}$ & $\begin{array}{c}B_{\mathrm{o}} \times 10^{-16} \\
{[\mathrm{SI}]}\end{array}$ \\
\hline \multicolumn{5}{c}{ 3. For $n$-propanol } \\
\hline 0.00 & 1.3429 & 1.3360 & 998.4 & 1.00 & 4.21 \\
0.03 & 1.3445 & 1.3376 & 992.4 & 1.22 & 5.14 \\
0.06 & 1.3461 & 1.3392 & 986.3 & 1.31 & 5.52 \\
0.10 & 1.3480 & 1.3411 & 979.0 & 1.36 & 5.57 \\
0.12 & 1.3493 & 1.3423 & 974.1 & 1.39 & 5.85 \\
0.19 & 1.3531 & 1.3461 & 959.5 & 1.50 & 6.32 \\
0.24 & 1.3557 & 1.3486 & 949.8 & 1.52 & 6.40 \\
0.28 & 1.3582 & 1.3512 & 940.1 & 1.55 & 6.53 \\
0.37 & 1.3633 & 1.3562 & 920.7 & 1.58 & 6.66 \\
0.45 & 1.3684 & 1.3613 & 901.2 & 1.61 & 6.78 \\
0.53 & 1.3757 & 1.3663 & 881.8 & 1.66 & 6.99 \\
0.60 & 1.3786 & 1.3713 & 862.3 & 1.69 & 7.12 \\
0.67 & 1.3836 & 1.3764 & 842.9 & 1.68 & 7.08 \\
0.74 & 1.3887 & 1.3814 & 823.4 & 1.67 & 7.03 \\
0.80 & 1.3938 & 1.3865 & 804.0 & 1.66 & 6.99 \\
\hline \multicolumn{6}{|c}{4. For acetone } \\
\hline 0.00 & 1.3392 & 1.3229 & 998.4 & 1.00 & 4.21 \\
0.01 & 1.3398 & 1.3335 & 996.3 & 1.13 & 4.76 \\
0.025 & 1.3406 & 1.3343 & 993.2 & 1.18 & 4.97 \\
0.05 & 1.3420 & 1.3356 & 988.0 & 1.35 & 5.69 \\
0.1 & 1.3449 & 1.3383 & 977.6 & 1.45 & 6.11 \\
0.15 & 1.3477 & 1.3410 & 967.1 & 1.62 & 6.82 \\
0.19 & 1.3505 & 1.3437 & 956.7 & 1.83 & 7.71 \\
0.24 & 1.3533 & 1.3464 & 946.3 & 1.97 & 8.30 \\
0.32 & 1.3589 & 1.3518 & 925.5 & 2.31 & 9.73 \\
0.37 & 1.3618 & 1.3545 & 915.0 & 2.45 & 10.3 \\
0.41 & 1.3646 & 1.3572 & 904.6 & 2.48 & 10.5 \\
0.45 & 1.3674 & 1.3599 & 894.2 & 2.56 & 10.8 \\
0.52 & 1.3730 & 1.3653 & 873.4 & 2.65 & 11.2 \\
0.60 & 1.3787 & 1.3707 & 852.5 & 2.68 & 11.3 \\
0.67 & 1.3843 & 1.3761 & 831.7 & 2.72 & 11.5 \\
0.73 & 1.3899 & 1.3815 & 810.8 & 2.57 & 10.8 \\
0.79 & 1.3956 & 1.3869 & 790.0 & 2.41 & 10.2 \\
\end{tabular}


TABLE II

The microscopic parameters calculated for the aqueous solutions studied from OKE results [SI].

\begin{tabular}{c|c|c|c|c|c}
\hline \hline $\begin{array}{c}\text { Aqueous } \\
\text { solution }\end{array}$ & $\begin{array}{c}\text { Partial } \\
\text { volume } \\
\nu \times 10^{-29}\end{array}$ & $\begin{array}{c}\text { Specific Kerr } \\
\text { constant } \\
B_{\mathrm{sp}} \times 10^{-19}\end{array}$ & $\begin{array}{c}\text { Anisotropic } \\
\text { factor } \\
\left(g_{1}-g_{2}\right)\end{array}$ & $\begin{array}{c}\text { Optical } \\
\text { anisotropy } \\
\delta \alpha \times 10^{-38}\end{array}$ & $\begin{array}{c}\text { Orientation } \\
\text { function } \\
f \times 10^{-19}\end{array}$ \\
\hline methanol & 6.75 & -1.38 & 0.57 & 0.43 & 10.7 \\
ethanol & 8.9 & 0.69 & 0.37 & 0.34 & 8.63 \\
propanol & 12.4 & 2.69 & 0.60 & 0.8 & 20.5 \\
acetone & 12.2 & 9.03 & 1.11 & 1.51 & 37.6
\end{tabular}

For methanol aqueous solutions the values of the anisotropic factor, optical anisotropy and orientation function were greater than for ethanol aqueous solutions. This fact can be explained by the ability of methanol to be associated with water via hydrogen bonds to a greater extent than ethanol.

TABLE III

The values of refractive indices $n_{\mathrm{a}}$ and $n_{\mathrm{i}}$, relative and absolute Kerr constant $B_{\mathrm{r}}$ and $B_{\mathrm{o}}$ as functions of temperature for $50 \% \mathrm{v} / \mathrm{v}$ aqueous solutions.

\begin{tabular}{c|c|c|c|c}
\hline \hline $\begin{array}{c}T \\
{[\mathrm{~K}]}\end{array}$ & $n_{\mathrm{a}}$ & $n_{\mathrm{i}}$ & $\begin{array}{c}B_{\mathrm{r}} \\
\text { to } \mathrm{H}_{2} \mathrm{O}\end{array}$ & $\begin{array}{c}B_{\mathrm{o}} \times 10^{-16} \\
{[\mathrm{SI}]}\end{array}$ \\
\hline \multicolumn{4}{|c}{ 1. For ethanol } \\
\hline 275.5 & 1.3691 & 1.3619 & 0.20 & 0.84 \\
277.5 & 1.3684 & 1.3612 & 0.25 & 1.05 \\
281.0 & 1.3672 & 1.3601 & 0.47 & 1.98 \\
282.0 & 1.3669 & 1.3598 & 0.50 & 2.11 \\
284.0 & 1.3662 & 1.3592 & 0.59 & 2.49 \\
287.0 & 1.3652 & 1.3582 & 0.80 & 3.37 \\
289.5 & 1.3645 & 1.3575 & 0.87 & 3.66 \\
291.0 & 1.3638 & 1.3569 & 0.97 & 4.09 \\
293.0 & 1.3632 & 1.3563 & 1.04 & 4.38 \\
296.0 & 1.3621 & 1.3554 & 1.25 & 5.27 \\
298.5 & 1.3613 & 1.3546 & 1.39 & 5.86 \\
301.5 & 1.3603 & 1.3536 & 1.47 & 6.19 \\
304.0 & 1.3594 & 1.3528 & 1.48 & 6.23 \\
308.0 & 1.3581 & 1.3515 & 1.60 & 6.74
\end{tabular}


TABLE III (cont.)

\begin{tabular}{c|c|c|c|c}
\hline \hline $\begin{array}{c}T \\
{[\mathrm{~K}]}\end{array}$ & $n_{\mathrm{a}}$ & $n_{\mathrm{i}}$ & $\begin{array}{c}B_{\mathrm{r}} \\
\text { to } \mathrm{H}_{2} \mathrm{O}\end{array}$ & $\begin{array}{c}B_{\circ} \times 10^{-16} \\
{[\mathrm{SI}]}\end{array}$ \\
\hline \multicolumn{4}{|c}{ 2. For acetone } \\
\hline 283.0 & 1.3716 & 1.3642 & 2.05 & 8.63 \\
285.0 & 1.3707 & 1.3634 & 2.19 & 9.22 \\
287 & 1.3699 & 1.3626 & 2.21 & 9.31 \\
289.0 & 1.3690 & 1.3618 & 2.28 & 9.60 \\
291.0 & 1.3682 & 1.3610 & 2.37 & 9.98 \\
293.0 & 1.3672 & 1.3600 & 2.34 & 9.86 \\
295.0 & 1.3665 & 1.3593 & 2.56 & 10.08 \\
297.5 & 1.3654 & 1.3582 & 2.77 & 11.7 \\
300.0 & 1.3644 & 1.3573 & 2.80 & 11.8 \\
302.0 & 1.3636 & 1.3565 & 2.90 & 12.2 \\
304.5 & 1.3626 & 1.3554 & 3.04 & 12.8 \\
307.5 & 1.3613 & 1.3542 & 3.22 & 13.6 \\
309.0 & 1.3607 & 1.3536 & 3.41 & 13.9 \\
310.5 & 1.3600 & 1.3530 & 3.42 & 14.4 \\
312.0 & 1.3594 & 1.3524 & 3.50 & 14.7 \\
314.0 & 1.3586 & 1.3516 & 3.62 & 15.3 \\
\hline \multicolumn{5}{|c}{3. For $n$-propanol } \\
\hline 275.5 & 1.3691 & 1.3619 & 0.20 & 0.84 \\
277.5 & 1.3684 & 1.3612 & 0.25 & 1.05 \\
281.0 & 1.3672 & 1.3601 & 0.47 & 1.98 \\
282.0 & 1.3669 & 1.3598 & 0.50 & 2.11 \\
284.0 & 1.3662 & 1.3592 & 0.59 & 2.49 \\
287.0 & 1.3652 & 1.3582 & 0.80 & 3.37 \\
289.5 & 1.3645 & 1.3575 & 0.87 & 3.66 \\
291.0 & 1.3638 & 1.3569 & 0.97 & 4.09 \\
293.0 & 1.3632 & 1.3563 & 1.04 & 4.38 \\
296 & 1.3621 & 1.3554 & 1.25 & 5.27 \\
298.5 & 1.3613 & 1.3546 & 1.39 & 5.86 \\
301.5 & 1.3603 & 1.3536 & 1.47 & 6.19 \\
304.0 & 1.3594 & 1.3528 & 1.48 & 6.23 \\
308 & 1.3581 & 1.3515 & 1.60 & 6.74 \\
& & & \\
\hline
\end{tabular}






Fig. 3. Temperature dependence of the absolute values of optical Kerr constant for the aqueous solutions studied.

We also studied the temperature dependence of the optically induced birefringence for $50 \%$ concentration aqueous solution of ethanol, $n$-propanol and acetone in the temperature range from $283 \mathrm{~K}$ to $315 \mathrm{~K}$. The heating system was thermostated with an accuracy of $\pm 0.05 \mathrm{~K}$, using a circulating ultrathermostat. The value of optical Kerr constant $B$ and the refractive indices $n_{\mathrm{a}}$ and $n_{\mathrm{i}}$ were determined in the same temperature range from $283 \mathrm{~K}$ to $315 \mathrm{~K}$ (Table III). Figure 3 presents changes of the optical Kerr constant with temperature for these aqueous solutions.

An analysis of the temperature dependence of the optical Kerr constant leads to the following conclusions:

- For ethanol and acetone (classified as dipolar aprotic liquids), when mixed with water $(50 \% \mathrm{v} / \mathrm{v}$ of solute) the distribution of their molecules becomes less sensitive to the influence of optical field with increasing temperature due to the solute-solvent interactions. Accordingly, the $B_{\circ}$ values changed linearly with temperature as shown in Fig. 3 which agrees with the theoretical predictions.

- Different behaviour of the temperature dependence of optically induced birefringence in the case of $n$-propanol aqueous solution can be explained by the cooperative nature of $n$-propanol molecules interactions. The strength of these cooperative interactions decreases with increasing temperature. In this system this process compensates the destructive temperature influence on the optical Kerr effect. 


\section{Acknowledgments}

This work was supported by the Research Project PB 1122/P3/93/04.

\section{References}

[1] M.I. Shakparonov, Research Methods of Molecular Thermal Motions and Structure of Liquids, Nauka, Moskva 1963 (in Russian).

[2] R. Patz, M.T. Rätzsch, Z. Phys. Chem. 265, 724 (1984).

[3] W. Pyzuk, H. Majgier-Baranowska, J. Zioło, Chem. Phys. 59, 111 (1981).

[4] M.V. Khashchina, P.L. Pakohomov, V.V. Prezhdo, Dokl. Akad. Nauk SSSR 250, 146 (1980).

[5] R. Piazza, V. Degiorgio, T. Bellini, J. Opt. Soc. Am. B 3, 1642 (1986).

[6] D.G. Tyson, B.R. Jennings, J. Phys. D, Appl. Phys. 24, 645 (1991).

[7] P.P. Ho, R.R. Alfano, Phys. Rev. A 20, 2170 (1979).

[8] C. Kalpouzos, Dale Mc Morrow, W.T. Lotshaw, G.A. Kenney-Wallace, Chem. Phys. Lett. 150, 138 (1988).

[9] H.-St. Albrecht, P. Heizt, J. Kleinschmidt, D. van Lap, T. Schröder, Appl. Phys. B 55, 362 (1992).

[10] Z. Błaszczak, M. Farhoud, in: Laser Study of Macroscopic Biosystems, Ed. E.J. Jouko Korppi-Tommola, Proc. SPIE, 1992, p. 12.

[11] M. Farhoud, Z. Błaszczak, Sh. Ramadan, Indian J. Pure Appl. Phys. 32, 517 (1994).

[12] N.J. Harrison, B.R. Jennings, J. Appl. Phys. 6, 8076 (1973).

[13] Z. Błaszczak, M. Farhoud, J. Chem. Soc. Faraday Trans. 90, 2455 (1994).

[14] A. Peterlin, H.A. Stuart, Z. Phys. 112, 129 (1939).

[15] S. Kielich, Acta Phys. Pol. A 37, 719 (1970).

[16] Z. Błaszczak, Acta Phys. Pol. A 68, 629 (1985).

[17] Z. Błasźczak, A. Dobek, A. Patkowski, Acta Phys. Pol. A 45, 269 (1974).

[18] Z. Błaszczak, Opt. Commun. 58, 439 (1986).

[19] Z. Błaszczak, P. Gauden, Opt. Commun. 94, 110 (1992). 